\title{
Edge states in quantum Hall effect in graphene
}

\section{(Review Article)}

\author{
V.P. Gusynin ${ }^{1}$, V.A. Miransky ${ }^{2}$ * , S.G. Sharapov ${ }^{3}$, and I.A. Shovkovy ${ }^{3} *$ \\ ${ }^{1}$ Bogolyubov Institute for Theoretical Physics, 03680, Kiev, Ukraine \\ E-mail: vgusynin@bitp.kiev.ua \\ ${ }^{2}$ Department of Applied Mathematics, University of Western Ontario, London, Ontario N6A 5B7, Canada \\ ${ }^{3}$ Department of Physics, Western Illinois University, Macomb, IL 61455, USA
}

Received February 15, 2008

\begin{abstract}
We review recent results concerning the spectrum of edge states in the quantum Hall effect in graphene. In particular, a special attention is payed to the derivation of the conditions under which gapless edge states exist in the spectrum of graphene with zigzag and armchair edges. We find that in the case of a half-plane or a ribbon with a zigzag edges, there are gapless edge states only when a spin gap dominates over a Dirac mass gap. In the case of a half-plane with an armchair edge, the existence of the gapless edge states depends on the specific type of Dirac mass gaps. The implications of these results for the dynamics in the quantum Hall effect in graphene are discussed.
\end{abstract}

PACS: 73.43.Cd Theory and modeling;

71.70.Di Landau levels;

81.05.Uw Carbon, diamond, graphite.

Keywords: graphene, quantum Hall effect, Dirac mass gap.

Contents

1. Introduction. . . . . . . . . . . . . . . . . . . . . . . . . . 999

2. Model with dynamical gaps . . . . . . . . . . . . . . . . . . . . 9995

3. Dirac equation in an external magnetic field . . . . . . . . . . . . . . . . 996

4. Edge states for a half-plane with a zigzag edge . . . . . . . . . . . . . . 9997

5. Edge states for a ribbon with zigzag edges . . . . . . . . . . . . 1000

6. Edge states for a half-plane with an armchair edge . . . . . . . . . . . . . 1002

7. Discussion. . . . . . . . . . . . . . . . . . . . . . . . . 1004

References . . . . . . . . . . . . . . . . . . . . 1005

\section{Introduction}

A graphite monolayer, or graphene, has become a new exciting topic in physics of two dimensional electronic systems [1-3]. A qualitatively new feature of graphene is that its low energy quasiparticles are described by a relativistic $2+1$ dimensional Dirac theory [4-6]. The spinor structure of the corresponding wave functions is a consequence of the honeycomb lattice structure of graphene with two carbon atoms per unit cell. When a magnetic field is applied, noninteracting Dirac quasiparticles occupy the Landau levels (LLs) with the following energies:

$$
E_{n}= \pm \sqrt{2 n \hbar v_{F}^{2}|e B| / c} \approx \pm 424 \sqrt{n} \sqrt{B[\mathrm{~T}]} \mathrm{K},
$$

with $n=0,1,2, \ldots$ Here $B$ is the value of the magnetic field orthogonal to the graphene's plane and $v_{F} \approx 10^{6} \mathrm{~m} / \mathrm{s}$ is the Fermi velocity.

* On leave from Bogolyubov Institute for Theoretical Physics, 03680, Kiev, Ukraine. 
Several anomalous properties of graphene are attributed to the presence of the lowest Landau level (LLL), i.e., the $n=0$ state in spectrum (1), whose energy is independent of the field strength. For example, the anomaly manifests itself as the phase shift of $\pi$ in the quantum magnetic oscillations of the diagonal conductivity. This phase shift can be understood theoretically by using either the semiclassical quantization condition for quasiparticles with a linear dispersion [7], or a microscopic calculation for both massless and massive Dirac fermions $[8,9]$. In the Hall conductivity, the anomaly results in an unconventional integer quantum Hall $(\mathrm{QH})$ effect with the plateaus at the filling factors $v= \pm 4(n+1 / 2)$ [10-13]. These and other distinct properties of graphene allow to identify unambiguously the Dirac nature of quasiparticles in experiments $[14,15]$.

While many unusual properties of graphene can be explained using the framework of a noninteracting Dirac theory, the quasiparticle interactions are not negligible. In fact, they are responsible for the appearance of additional QH plateaus with the filling factors $v=0, \pm 1, \pm 4$ that were first reported in Ref. 16 in the case of sufficiently strong magnetic fields, $B \gtrsim 20 \mathrm{~T}$ (see also Refs. 17-20).

Recently we proposed a dynamical mechanism [21], based on the phenomenon of the magnetic catalysis [22], that could explain the $v=0$ and $v= \pm 1$ plateaus in the Hall conductivity of graphene [16]. The subsequent experiments $[17,18]$ have revealed several additional features of the $v=0$ and $v= \pm 1$ plateaus that seem to require modifications of the scenario in Ref. 21. Among them, the most important is a rather peculiar dissipative nature of the diagonal transport at the $v=0$ plateau. This seems to suggest that the origin of the $v=0$ plateau is associated with a spin rather than mass gap $[17,23]$. This conclusion is supported by the fact that the activation energy at the $v=0$ plateau is vanishing $[17,18]$. Additionally, the diagonal transport is suggested to be dominated by gapless edge states which should exist when the lowest Landau level is split by a large spin gap $[17,23]$.

Concerning the $v= \pm 1$ plateaus, the measurements of the thermal activation energy $\Delta E(v= \pm 1)$ point at its connection with orbital dynamics. Indeed, the activation energy depends only on the perpendicular component of the magnetic field $[17,18]$. The dynamical nature of the $v= \pm 1$ plateaus is also suggested by the fact that $\Delta E(v= \pm 1)$ is proportional to $\sqrt{B}[17,19]$.

Note that, in contrast, the $v= \pm 4$ plateaus can be consistently associated with the Zeeman splitting of the $n=1$ Landau level. The corresponding activation energy $\Delta E(v= \pm 4)$ depends linearly on the total magnetic field and has the same magnitude as the Zeeman energy $[17,18]$,

$$
E_{Z}=\frac{g_{L}}{2} \mu_{B} B \simeq 0.67 B[\mathrm{~T}] \mathrm{K},
$$

where $\mu_{B}=e \hbar /(2 m c)$ is the Bohr magneton and $g_{L} \simeq 2$ is the Lande factor in graphene.

Theoretically, the $v=0$ and $v= \pm 1$ plateaus come from lifting the approximate degeneracy of four sublevels at LLL. The degeneracy is a consequence of the «flavor» $U(4)$ symmetry of the low-energy continuum description of graphene in absence of the Zeeman interaction. This symmetry operates in the space of two sublattice-valley and two spin degrees of freedom. If it is accepted that the $v=0$ plateau is due to a spin gap, then the $v= \pm 1$ plateaus should result from breaking the valley-sublattice symmetry. This seems to be in agreement with the observations in Ref. 18.

There are essentially two approaches that consider various possibilities of breaking the approximate $U(4)$ symmetry of graphene (for a brief review, see Ref. 24):

i) The quantum Hall ferromagnetism (QHF) scenario [25-27] that exploits an analogy between the four-fold degeneracy of LLs in graphene, associated with the $U(4)$ symmetry, and the $S U(4)$ ferromagnetism previously studied in the bilayer quantum Hall systems [28]. In this scenario the QH plateaus with all integer values of the filling factor $v$ occur in sufficiently clean samples. The QHF order parameters are described by the densities of conserved charges connected with the diagonal generators of the $S U(4) \subset U(4)$ symmetry group.

ii) The magnetic catalysis (MC) scenario [21,29-31] uses the idea of a spontaneous symmetry breaking due to the exciton (chiral) condensation [22,32-34]. Such a condensation produces a nonzero Dirac mass term in the low-energy theory of graphene. (Note that originally the magnetic catalysis scenario in graphene was motivated by the early experiments in highly oriented pyrolytic graphite [35].)

As emphasized in Ref. 21, the plateau $v=0$ could appear due to either an enhanced spin gap or a mass term. An enhanced spin gap breaks the approximate $U(4)$ symmetry down to the $U(2)_{-} \times U(2)_{+}$subgroup which operates in the sublattice-valley space and does not mix spin-up $(s=+)$ and spin-down $(s=-)$ states. A nonzero Dirac mass term breaks the symmetry down to another $U(2) \times U(2)^{\prime}$ subgroup, operating in the spin space. Either of them is sufficient to partially lift the four-fold degeneracy of the LLL that is needed in the $v=0 \mathrm{QH}$ state. The structure of the energy sublevels at LLL in the case of a nonzero spin gap is illustrated in the left panel of Fig. 1.

In order to explain the $v= \pm 1 \mathrm{QH}$ plateaus two different order parameters are required. (Note that the choice of two order parameters with given symmetry properties is not unique [36].) This should be evident already from the symmetry arguments alone. For example, the simplest possible structure of the energy sublevels for the $v=+1$ state is shown in the right panel of Fig. 1. The corresponding splitting is possible only if the $U(2)_{-} \times U(2)_{+}$sym- 


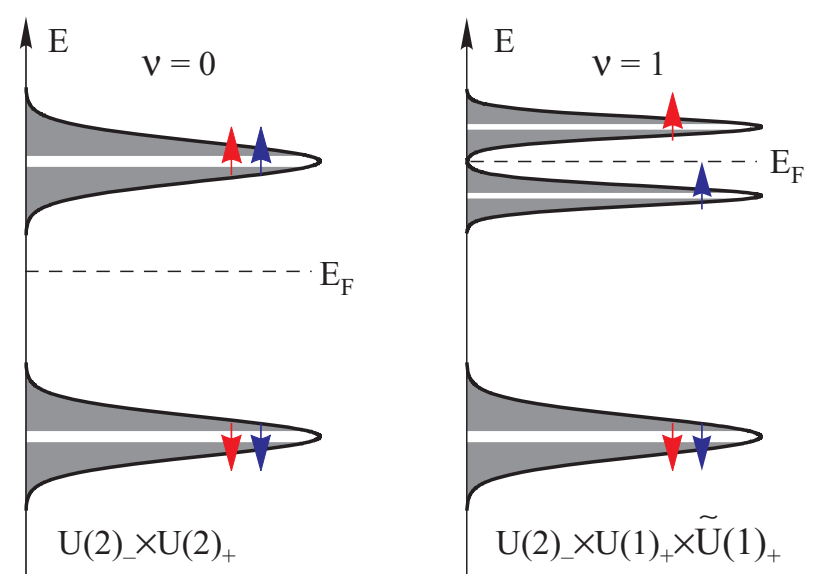

Fig. 1. Illustration of the lowest Landau level splitting needed to explain $v=0$ and $v=1$ plateaus in QHE in graphene.

metry is further reduced, e.g., at least down to the $U(2)_{-} \times U(1)_{+} \times \widetilde{U}(1)_{+}$subgroup. However, this would not be possible without having an additional order parameter that breaks the sublattice-valley symmetry, described by the simple Lie group $S U(2)_{+} \subset U(2)_{+}$.

An approach that combines both QHF and MC mechanisms in a unifying scheme was recently proposed in Ref. 36. By making use of a multi-parameter variational ansatz for the quasiparticle propagator, it was found that QHF $\left(\mu_{s}\right.$ and $\left.\widetilde{\mu}_{s}\right)$ and $\mathrm{MC}\left(\Delta_{s}\right.$ and $\left.\widetilde{\Delta}_{s}\right)$ order parameters necessarily coexist. In terms of symmetry, the order parameters of the first type, i.e., $\mu_{s}$ and $\Delta_{s}$ with nonequal values for $s= \pm$, break the $U(4)$ symmetry down to $U(2)_{-} \times U(2)_{+}$just like the Zeeman term. The order parameters of the other type, $\widetilde{\mu}_{s}$ and $\widetilde{\Delta}_{s}$, are triplets with respect to the $S U(2)_{s}$ group which is the largest nonabelian subgroup of the $U(2)_{s}$. Thus, when either $\widetilde{\mu}_{s}$ or $\widetilde{\Delta}_{s}$ has a nonzero vacuum expectation value, the symmetry $S U(2)_{s}$ is further broken down to $U(1)_{s}$.

In this paper we review the results of Refs. 37 and 38 that address the question of compatibility of the microscopic dynamics described in Ref. 36 with the gapless edge states, apparently needed for the description of the $v=0$ plateau $[17,18]$. The main conclusions are as follows. In the case of graphene on a half-plane or a finite width ribbon with zigzag edges [37,38], there are gapless edge states in the spectrum only when the spin gap dominates over the mass gap. In the case of graphene on a half-plane with an armchair edge [38], however, the existence of the gapless edge states depends on the specific type of the mass gaps. These results could have important consequences for understanding dynamics in the $\mathrm{QH}$ effect in graphene.

The paper is organized as follows. In Sec. 2 we present a model Lagrangian that captures the most general dynamical situation with QHF and MC order parameters, proposed in Ref. 36. The spectrum of the corresponding Dirac equation in an external magnetic field is analyzed in Sec. 3. The edge states for graphene on a half-plane with zigzag and armchair edges are considered in Secs. 4 and 6 , respectively. The edge states for graphene ribbon with zigzag edges are analyzed in Sec. 5. The main results of the paper are discussed in Sec. 7.

\section{Model with dynamical gaps}

The low-energy quasiparticles excitations in graphene are described in terms of a four-component Dirac spinors $\Psi_{s}^{T}=\left(\psi_{K_{+} A s}, \psi_{K_{+} B s}, \psi_{K_{-} B s}, \psi_{K_{-} A s}\right)$. Each spinor (with a given spin index $s= \pm$ ) combines the Bloch states on the two different sublattices ( $A$ and $B$ ) of the hexagonal graphene lattice and with momenta near the two inequivalent Dirac points $\left(K_{+}\right.$and $\left.K_{-}\right)$of the two-dimensional Brillouin zone. The quadratic part of low-energy Lagrangian density for quasiparticles can be written in a relativistic form,

$$
\begin{aligned}
& \mathcal{L}=\sum_{s= \pm} \hbar \bar{\Psi}_{s}(t, \mathbf{r})\left(i \gamma^{0} \partial_{t}+i v_{F} \gamma^{1} D_{x}+i v_{F} \gamma^{2} D_{y}\right) \Psi_{S}(t, \mathbf{r})+ \\
& +\mathcal{L}_{\text {mass }}+\sum_{s= \pm}\left(\mu_{s} \rho_{s}+\widetilde{\mu}_{s} \widetilde{\rho}_{s}\right)
\end{aligned}
$$

where $\bar{\Psi}_{s}=\Psi_{s}^{\dagger} \gamma^{0}$ is the Dirac conjugated spinor and the operators $\rho_{s} \equiv \Psi_{s} \gamma^{0} \Psi_{s}$ and $\widetilde{\rho}_{s} \equiv \bar{\Psi}_{s} \gamma^{0} \gamma^{5} \Psi_{s}$ are densities of conserved charges connected with the chemical potentials $\mu_{s}$ and $\widetilde{\mu}_{s}$, respectively. Notice that here the Fermi velocity $v_{F} \approx c / 300$ plays the role of the speed of light. The orbital effect of a perpendicular magnetic field $\mathbf{B}=\nabla \times \mathbf{A}$ is included via the covariant derivative $D_{i}=\partial_{i}+(i e / \hbar c) A_{i}$ where $i=x, y$ and $-e<0$ is the electron charge. Here we assume that the vector potential is taken in the Landau gauge: $A_{x}=-B y$ and $A_{y}=0$, where $B$ is the magnitude of the magnetic field. The mass term $\mathcal{L}_{\text {mass }}$ is defined below.

The $4 \times 4$ matrices $\gamma^{v}$ furnish a reducible representation of the Dirac algebra. Here, we use the following representation:

$$
\gamma^{0}=\widetilde{\tau}_{1} \otimes \tau_{0}, \gamma^{i}=-i \tilde{\tau}_{2} \otimes \tau_{i},
$$

where the Pauli matrices $\widetilde{\tau}_{i}, \tau_{i}$ (as well as the $2 \times 2$ unit matrices $\left.\widetilde{\tau}_{0}, \tau_{0}\right)$ act on the valley $\left(K_{+}, K_{-}\right)$and sublattice $(A, B)$ indices, respectively. This representation is derived from a tight-binding model for graphene [39]. It is particularly convenient for our purposes in this study because it provides a simple form of the boundary conditions at zigzag and armchair edges. As follows from defnition (4), the $\gamma$ matrices satisfy the usual anticommutation relations $\left\{\gamma^{\mu}, \gamma^{\nu}\right\}=2 g^{\mu \nu}$ where $g^{\mu \nu}=\operatorname{diag}(1,-1,-1,-1)$. Since the matrix $\gamma^{5} \equiv i \gamma^{0} \gamma^{1} \gamma^{2} \gamma^{3}$ is diagonal,

$$
\gamma^{5}=\left(\begin{array}{cc}
I_{2} & 0 \\
0 & -I_{2}
\end{array}\right)
$$


this representation is conventionally called chiral. Note that the chirality here is identified with the valley index $\left(K_{+}\right.$or $\left.K_{-}\right)$[39].

The general expression for the mass term $\mathcal{L}_{\text {mass }}$ in the Lagrangian density may include singlet $\left(\Delta_{s}\right)$ as well as triplet $\left(\widetilde{\Delta}_{S}\right)$ contributions with respect to the valley symmetry group $S U(2)_{s}$. The appearance of the mass term can be attributed, for example, to the $\mathrm{MC}$ mechanism. In the representation used here, its explicit form reads

$$
\mathcal{L}_{\text {mass }}=\sum_{s= \pm} \bar{\Psi}_{s}(t, \mathbf{r})\left(\Delta_{s} \gamma^{3} \gamma^{5}-\widetilde{\Delta}_{s} \gamma^{3}\right) \Psi_{s}(t, \mathbf{r}) .
$$

Under the time reversal symmetry, the operators associated with the mass parameters $\Delta_{S}$ and $\widetilde{\Delta}_{S}$ are odd and even, respectively. Concerning the triplet mass term $\widetilde{\Delta}_{s} \bar{\Psi}_{s} \gamma^{3} \Psi_{s}$, it can be also written in other equivalent forms, e.g., as $\widetilde{\Delta}_{S} \bar{\Psi}_{s} i \gamma^{5} \Psi_{S}$ or $\widetilde{\Delta}_{S} \bar{\Psi}_{S} \Psi_{s}$ [40]. The latter, in particular, is the usual Dirac mass term. All of these repre- sentations are equivalent because they are related by transformations of the $S U(2)_{S}$ symmetry group. For our purposes, however, it is most convenient to use the form in Eq. (6) which, as we shall see below, has a simple interpretation in the tight binding model.

In Lagrangian density (3), the chemical potentials $\mu_{s}$ and $\tilde{\mu}_{s}$ allow to accommodate the QHF order parameters in the dynamical model of Ref. 36. Regarding the transformation properties of $\mu_{s}$ and $\widetilde{\mu}_{s}$ under the flavor symmetry, they are similar to those of $\Delta_{s}$ and $\widetilde{\Delta}_{s}$, respectively.

Before proceeding with further analysis, it is instructive to address the physics interpretation of the mass parameters and chemical potentials in more detail. To this end, let us write down the explicit expressions for the corresponding operators in the Lagrangian density in terms of separate Bloch components of the spinors,

$$
\begin{aligned}
& \widetilde{\Delta}_{s}: \bar{\Psi}_{s} \gamma^{3} \Psi_{s}=\psi_{K_{+} A s}^{\dagger} \psi_{K_{+} A s}+\psi_{K_{-} A s}^{\dagger} \psi_{K_{-} A s}-\psi_{K_{+} B s}^{\dagger} \psi_{K_{+} B s}-\psi_{K_{-} B s}^{\dagger} \psi_{K_{-} B s}, \\
& \Delta_{s}: \bar{\Psi}_{s} \gamma^{3} \gamma^{5} \Psi_{s}=\psi_{K_{+} A s}^{\dagger} \psi_{K_{+} A s}-\psi_{K_{-} A s}^{\dagger} \psi_{K_{-} A s}-\psi_{K_{+} B s}^{\dagger} \psi_{K_{+} B s}+\psi_{K_{-} B s}^{\dagger} \psi_{K_{-} B s}, \\
& \widetilde{\mu}_{s}: \bar{\Psi}_{s} \gamma^{0} \gamma^{5} \Psi_{s}=\psi_{K_{+} A s}^{\dagger} \psi_{K_{+} A s}-\psi_{K_{-} A s}^{\dagger} \psi_{K_{-} A s}+\psi_{K_{+} B s}^{\dagger} \psi_{K_{+} B s}-\psi_{K_{-} B s}^{\dagger} \psi_{K_{-} B s}, \\
& \mu_{s}: \bar{\Psi}_{s} \gamma^{0} \Psi_{s}=\psi_{K_{+} A s}^{\dagger} \psi_{K_{+} A s}+\psi_{K_{-} A s}^{\dagger} \psi_{K_{-} A s}+\psi_{K_{+} B s}^{\dagger} \psi_{K_{+} B s}+\psi_{K_{-} B s}^{\dagger} \psi_{K_{-} B s} .
\end{aligned}
$$

Here the operators on the right hand side are linear combinations of the electron densities at specified valleys $\left(K_{+}\right.$ or $K_{-}$) and sublattices ( $A$ or $B$ ). These operators enter into the Lagrangian density together with the parameters $\Delta_{s}$, $\widetilde{\Delta}_{s}, \mu_{s}$, and $\widetilde{\mu}_{s}$ which play the role of Lagrange multipliers. Therefore, the values of the masses and chemical potentials control the relative concentrations of electrons at different valleys and sublattices. They are determined from the gap equations for Dirac quasiparticles [36].

As seen from Eq. (7), the triplet Dirac mass $\widetilde{\Delta}_{S}$ is related to the density imbalance between the $A$ and $B$ sublattices. Its spontaneous generation leads to a state with a charge density wave [21,29-32]. It has also been suggested [41] that this kind of Dirac mass can be induced in graphene by placing it on a top of an appropriate substrate that breaks the sublattice symmetry. Recent ARPES measurements at $B=0$ show an evidence for a finite value of the gap $\widetilde{\Delta}$ in epitaxial graphene grown on $\mathrm{SiC}$ [42]. If the values of the masses are nonequal for different spins $s= \pm$, an admixture of an antiferromagnetic wave also develops in the ground state [29]. A mixed density imbalance at two valleys and two sublattices is controlled by the singlet mass $\Delta_{s}$, see Eq. (8). Similarly, the chemical potential $\widetilde{\mu}_{s}$ is connected with the density imbalance between the two valleys. At last, $\mu_{s}$ is the usual chemical po- tential related to the total density of electrons with a given spin.

\section{Dirac equation in an external magnetic field}

In this section we study the spectrum of the low-energy quasiparticles in the model of graphene with the most general set of parameters $\Delta_{s}, \widetilde{\Delta}_{s}, \mu_{s}$, and $\widetilde{\mu}_{s}$. The corresponding Dirac equation takes the following form:

$$
\begin{aligned}
& {\left[i \gamma^{0} \hbar \partial_{t}+i \hbar v_{F} \gamma^{1} D_{x}+i \hbar v_{F} \gamma^{2} D_{y}+\right.} \\
& \left.+\mu \gamma^{0}+\widetilde{\mu} \gamma^{0} \gamma^{5}+\Delta \gamma^{3} \gamma^{5}-\widetilde{\Delta} \gamma^{3}\right] \Psi(t, \mathbf{r})=0 .
\end{aligned}
$$

For brevity of notation, the spin index is omitted here and below. For the energy eigenvalue solutions $\Psi(t, \mathbf{r})=$ $=\exp (-i E t / \hbar) \Psi(\mathbf{r})$ the equation reduces to

$$
\begin{aligned}
& {\left[\hbar v_{F}\left(-\alpha_{1} i D_{x}-\alpha_{2} i D_{y}\right)-\right.} \\
& \left.-\mu-\widetilde{\mu} \gamma^{5}-i \Delta \gamma^{1} \gamma^{2}+\widetilde{\Delta} \alpha_{3}\right] \Psi(\mathbf{r})=E \Psi(\mathbf{r}),
\end{aligned}
$$

where the $\alpha$ matrices are

$$
\alpha_{i}=\gamma^{0} \gamma^{i}=\left(\begin{array}{cc}
\sigma_{i} & 0 \\
0 & -\sigma_{i}
\end{array}\right)
$$

Using the representation for the $\gamma$ matrices in Eq. (4), we can rewrite the Dirac equation in components as follows: 


$$
\begin{gathered}
\left(\begin{array}{cc}
-\mu^{(+)}-\Delta^{(-)} & -\hbar v_{F}\left(i D_{x}+D_{y}\right) \\
-\hbar v_{F}\left(i D_{x}-D_{y}\right) & -\mu^{(+)}+\Delta^{(-)}
\end{array}\right)\left(\begin{array}{l}
\psi_{A K_{+}} \\
\psi_{B K_{+}}
\end{array}\right)=E\left(\begin{array}{l}
\psi_{A K_{+}} \\
\psi_{B K_{+}}
\end{array}\right), \\
\left(\begin{array}{cc}
-\mu^{(-)}-\Delta^{(+)} & \hbar v_{F}\left(i D_{x}+D_{y}\right) \\
\hbar v_{F}\left(i D_{x}-D_{y}\right) & -\mu^{(-)}+\Delta^{(+)}
\end{array}\right)\left(\begin{array}{l}
\psi_{B K_{-}} \\
\psi_{A K_{-}}
\end{array}\right)=E\left(\begin{array}{c}
\psi_{B K_{-}} \\
\psi_{A K_{-}}
\end{array}\right) .
\end{gathered}
$$

Here we introduced the shorthand notation: $\mu^{( \pm)} \equiv \mu \pm \widetilde{\mu}$ and $\Delta^{( \pm)} \equiv \Delta \pm \widetilde{\Delta}$. As we see, the equations for different valleys decouple. This is a very useful property that simplifies the analysis considerably. In each of the two decoupled sets of equations, we can express the $B$ components in terms of the $A$ components of the spinors,

$$
\begin{aligned}
& \psi_{B K_{+}}=-\frac{\hbar v_{F}\left(i D_{x}-D_{y}\right)}{E+\mu^{(+)}-\Delta^{(-)}} \psi_{A K_{+}}, \\
& \psi_{B K_{-}}=\frac{\hbar v_{F}\left(i D_{x}+D_{y}\right)}{E+\mu^{(-)}+\Delta^{(+)}} \psi_{A K_{-}} .
\end{aligned}
$$

Then, at $K_{+}$and $K_{-}$valleys, the two-component spinors can be written in the following form:

$$
\begin{aligned}
& \psi_{K_{+}}=A_{1}\left(\begin{array}{c}
\psi_{A K_{+}} \\
-\frac{\hbar v_{F}\left(i D_{x}-D_{y}\right)}{E+\mu^{(+)}-\Delta^{(-)}} \psi_{A K_{+}}
\end{array}\right), \\
& \psi_{K_{-}}=A_{2}\left(\begin{array}{c}
\frac{\hbar v_{F}\left(i D_{x}+D_{y}\right)}{E+\mu^{(-)}+\Delta^{(+)}} \psi_{A K_{-}} \\
\psi_{A K_{-}}
\end{array}\right) .
\end{aligned}
$$

Here the constants $A_{1,2}$ are determined by the normalization conditions

$$
\int d^{2} r \psi_{K_{ \pm}}^{\dagger}(\mathbf{r}, k, n) \psi_{K_{ \pm}}\left(\mathbf{r}, k^{\prime}, n^{\prime}\right)=\delta_{n, n^{\prime}} \delta\left(k-k^{\prime}\right),
$$

where $k, k^{\prime}$ and $n, n^{\prime}$ are the quantum numbers (e.g., the wavevector along $x$ or $y$ direction and the Landau level index) that characterize the eigenstates of Dirac quasiparticles in the magnetic field.

As follows from Eqs. (14) and (15), the $A$ components of the spinors satisfy the following second order differential equations

$$
\begin{aligned}
& \left(-l^{2} D_{x}^{2}-l^{2} D_{y}^{2}+1\right) \psi_{A K_{+}}=2 \lambda_{+} \psi_{A K_{+}}, \\
& \left(-l^{2} D_{x}^{2}-l^{2} D_{y}^{2}-1\right) \psi_{A K_{-}}=2 \lambda_{-} \psi_{A K_{-}} .
\end{aligned}
$$

Here we introduced the two dimensionless parameters $\lambda_{ \pm} \equiv\left[\left(E+\mu^{( \pm)}\right)^{2}-\left(\Delta^{(\mp)}\right)^{2}\right] / \varepsilon_{0}^{2}$, the Landau energy scale $\varepsilon_{0} \equiv \sqrt{2 \hbar v_{F}^{2}|e B| / c}$, and the magnetic length $l \equiv$ $\equiv \sqrt{\hbar c /|e B|}$.

In the Landau gauge $\left(A_{x}, A_{y}\right)=(-B y, 0)$, the differential equations in Eq. (21) do not depend explicitly on the $x$ coordinate and, therefore, the wave functions are plane waves in the $x$ direction,

$$
\begin{aligned}
& \psi_{A K_{+}}(\mathbf{r}, k)=\frac{1}{\sqrt{2 \pi l}} \mathrm{e}^{i k x} u_{+}(y, k), \\
& \psi_{B K_{+}}(\mathbf{r}, k)=\frac{1}{\sqrt{2 \pi l}} \mathrm{e}^{i k x} v_{+}(y, k), \\
& \psi_{A K_{-}}(\mathbf{r}, k)=\frac{1}{\sqrt{2 \pi l}} \mathrm{e}^{i k x} u_{-}(y, k), \\
& \psi_{B K_{-}}(\mathbf{r}, k)=\frac{1}{\sqrt{2 \pi l}} \mathrm{e}^{i k x} v_{-}(y, k),
\end{aligned}
$$

where the functions $u_{ \pm}(y, k)$ depend only on a single combination of the variables, $\xi=y / l-k l$, and satisfy the following equations:

$$
\left(\partial_{\xi}^{2}-\xi^{2} \mp 1+2 \lambda_{ \pm}\right) u_{ \pm}(\xi)=0 .
$$

In accordance with Eq. (18), the eliminated components $v_{ \pm}(y, k) \equiv v_{ \pm}(\xi)$ are given by

$$
v_{ \pm}(\xi)=\frac{\varepsilon_{0}\left(\partial_{\xi} \mp \xi\right) u_{ \pm}(\xi)}{\sqrt{2}\left(E+\mu^{( \pm)} \mp \Delta^{(\mp)}\right)} .
$$

In an infinite system without boundaries, normalizable solutions to Eqs. (23) are expressed in terms of the Hermite polynomials, $u(\xi), v(\xi) \propto \mathrm{e}^{-\xi^{2} / 2} H_{n}(\xi)$, provided the parameters $\lambda_{ \pm}$take nonnegative integer values, i.e.,

$$
\lambda_{ \pm}=n, \text { where } n=0,1,2, \ldots
$$

Note that the value of the energy $E=-\mu^{(+)}+\Delta^{(-)}$corresponds to a normalizable LLL state in $K_{+}$valley. For such a state, the apparent singularity in the $v_{+}(\xi)$ component of the wave function, see Eq. (24), is removed by a proper redefinition of the normalization constant. The same is not true, however, for the value of the energy $E=-\mu^{(-)}-\Delta^{(+)}$in the $K_{-}$valley. In fact, a direct analysis shows that the only $n=0$ state in $K_{+}$valley has energy $E=-\mu^{(+)}+\Delta^{(-)}$and resides solely on the $B$ sublattice, while the only $n=0$ state in $K_{\text {_ }}$ valley has energy $E=-\mu^{(-)}+\Delta^{(+)}$and resides solely on the $A$ sublattice.

\section{Edge states for a half-plane with a zigzag edge}

There exist many studies of edge states in graphene under various conditions [13,17,23,37,38,43-49]. Here we first consider a graphene monolayer on the half-plane $y>0$ with a zigzag edge parallel to the $x$ as shown in Fig. 2 [38]. To obtain the energy spectrum we need to supplement the differential equations for the $u_{ \pm}(y, k)$ and $v_{ \pm}(y, k)$ functions with suitable boundary conditions. Such conditions can be derived from the tight-binding model $[23,45,47]$. For example, for a zigzag edge parallel to the $x$ axis the wave function on $A$ atoms should vanish at $y=0$,

$$
u_{+}(y=0)=u_{-}(y=0)=0 \text {. }
$$




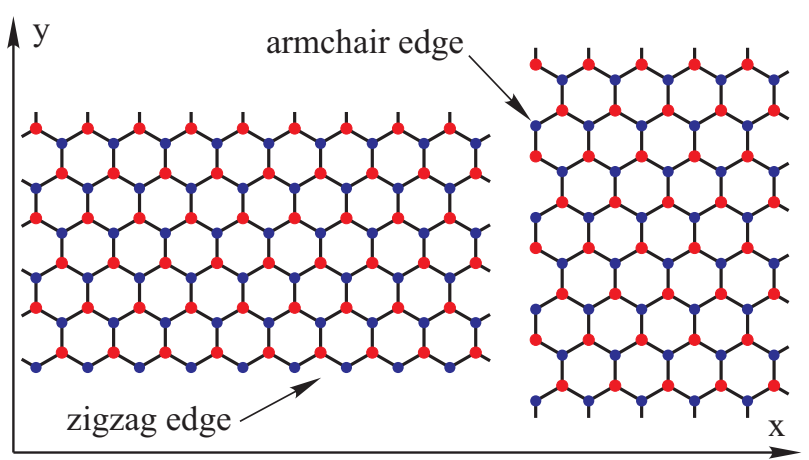

Fig. 2. Graphene lattice with zigzag and armchair edges.

The general solution to Eq. (23) is expressed in terms of the parabolic cylinder (Weber) functions $U(a, z)$ and $V(a, z)[50]$,

$$
\begin{aligned}
& u_{+}(\xi)=C_{1} \frac{E+\mu^{(+)}-\Delta^{(-)}}{\varepsilon_{0}} U\left(\frac{1-2 \lambda_{+}}{2}, \sqrt{2} \xi\right)+ \\
& +C_{2} V\left(\frac{1-2 \lambda_{+}}{2}, \sqrt{2} \xi\right) \\
& u_{-}(\xi)=C_{3} U\left(-\frac{1+2 \lambda_{-}}{2}, \sqrt{2} \xi\right)+ \\
& +C_{4} \frac{E+\mu^{(-)}+\Delta^{(+)}}{\varepsilon_{0}} V\left(-\frac{1+2 \lambda_{-}}{2}, \sqrt{2} \xi\right) .
\end{aligned}
$$

Here, for convenience of the further analysis, the integration constants $C_{1}$ and $C_{4}$ are introduced together with the additional factors $\left(E+\mu^{(+)}-\Delta^{(-)}\right) / \varepsilon_{0}$ and $\left(E+\mu^{(-)}+\Delta^{(+)}\right) / \varepsilon_{0}$, respectively.

In an infinite system without edges, the normalizable wave functions contain only the parabolic cylinder $U(a, z)$ functions which are bound at $z \rightarrow \pm \infty$, provided $a=-n-1 / 2$ and $n$ is a nonnegative integer. In this case, the following relation is valid: $U(-n-1 / 2, z)=$ $=2^{-n / 2} \mathrm{e}^{-z^{2} / 4} H_{n}(z / \sqrt{2})$, where $H_{n}(z)$ are the Hermite polynomials. Therefore, as stated in the previous section, the spectrum is given by $\lambda_{ \pm}=n$ where $n=0,1,2, \ldots$ (A special nature of LLL should be kept in mind: at $n=0$ there are only two rather than four possible energy eigenvalues that correspond to normalizable states.)

Using the following recurrent relations for parabolic cylinder functions [50]

$$
\begin{aligned}
& \left(\frac{d}{d z}+\frac{z}{2}\right) U(a, z)=-\left(a+\frac{1}{2}\right) U(a+1, z), \\
& \left(\frac{d}{d z}-\frac{z}{2}\right) U(a, z)=U(a-1, z), \\
& \left(\frac{d}{d z}+\frac{z}{2}\right) V(a, z)=V(a+1, z), \\
& \left(\frac{d}{d z}-\frac{z}{2}\right) V(a, z)=\left(a-\frac{1}{2}\right) V(a-1, z),
\end{aligned}
$$

and Eq. (24), we obtain the $v_{ \pm}(\xi)$ functions,

$$
\begin{aligned}
& v_{+}(\xi)=-C_{1} U\left(-\frac{1+2 \lambda_{+}}{2}, \sqrt{2} \xi\right)- \\
& -C_{2} \frac{E+\mu^{(+)}+\Delta^{(-)}}{\varepsilon_{0}} V\left(-\frac{1+2 \lambda_{+}}{2}, \sqrt{2} \xi\right), \\
& v_{-}(\xi)=C_{3} \frac{E+\mu^{(-)}-\Delta^{(+)}}{\varepsilon_{0}} U\left(\frac{1-2 \lambda_{-}}{2}, \sqrt{2} \xi\right)+ \\
& +C_{4} V\left(\frac{1-2 \lambda_{-}}{2}, \sqrt{2} \xi\right) .
\end{aligned}
$$

On a half-plane, normalizable wave functions are also given in terms of only $U(a, z)$ function which falls off exponentially as $z \rightarrow+\infty$, while the function $V(a, z)$ is growing exponentially in both directions $z \rightarrow \pm \infty$. Therefore, we must take $C_{2}=0$ and $C_{4}=0$. In contrast to the case of an infinite plane, on a half-plane there is no restriction for the parameter $a$ to be a negative half-integer.

Taking into account that $C_{2}=C_{4}=0$, the zigzag boundary conditions (26) lead to the following system of equations

$$
\begin{aligned}
& C_{1}\left(E+\mu^{(+)}-\Delta^{(-)}\right) D_{\lambda_{+}-1}(-\sqrt{2} k l)=0, \\
& C_{3} D_{\lambda_{-}}(-\sqrt{2} k l)=0 .
\end{aligned}
$$

Here we introduced another parabolic cylinder function, $D_{v}(z)$ [50], which is related to function $U(a, z)$ in a simple way:

$$
U(a, z)=D_{-a-1 / 2}(z) .
$$

There are two types of nontrivial solutions that satisfy boundary conditions (32). Firstly, taking $C_{1} \neq 0$ and $C_{3}=0$, we find that the equation for eigenvalues is reduced down to $E=-\mu^{(+)}+\Delta^{(-)}$or

$$
\text { I. } D_{\lambda_{+}-1}(-k l \sqrt{2})=0 \text {. }
$$

The solutions of this type have wave functions with a support only in $K_{+}$valley,

$$
\text { I. } \begin{aligned}
u_{+}(\xi) & =C_{1} \frac{E+\mu^{(+)}-\Delta^{(-)}}{\varepsilon_{0}} D_{\lambda_{+}-1}(\sqrt{2} \xi), \\
v_{+}(\xi) & =-C_{1} D_{\lambda_{+}}(\sqrt{2} \xi)
\end{aligned}
$$

and $u_{-}(\xi)=v_{-}(\xi)=0$. The other class of solutions is such that $C_{1}=0$ and $C_{3} \neq 0$, and the energy eigenvalues satisfy the following equation:

$$
\text { II. } D_{\lambda_{-}}(-k l \sqrt{2})=0 \text {. }
$$

The wave functions for this type solutions are nonvanishing only in $K_{\text {_ }}$ valley, i.e., 


$$
\text { II. } \begin{aligned}
u_{-}(\xi) & =C_{3} D_{\lambda_{-}}(\sqrt{2} \xi), \\
v_{-}(\xi) & =C_{3} \frac{E+\mu^{(-)}-\Delta^{(+)}}{\varepsilon_{0}} D_{\lambda_{-}-1}(\sqrt{2} \xi),
\end{aligned}
$$

and $u_{+}(\xi)=v_{+}(\xi)=0$. By making use of the general properties of the parabolic cylinder function $D_{v}(z)$, we can understand some qualitative features of the energy spectrum even without solving the equations numerically. To this end, we need to know only that, for real $v$ and $z$, the function $D_{v}(z)$ has no real zeros when $v$ is negative, and has exactly $[v+1]$ real zeros when $v$ is nonnegative [51]. Here $[v+1]$ denotes the integer part of $v+1$. In view of this property, the necessary condition for Eq. (34) to be satisfied is $\lambda_{+} \geq 1$. By including also the possibility of the dispersionless mode, determined by $E=-\mu^{(+)}+\Delta^{(-)}$, we see that the complete spectrum in valley $K_{+}$(solutions of type I) has the following general structure:

$$
\begin{aligned}
& E_{0}(k)=-\mu^{(+)}+\Delta^{(-)}, \\
& E_{n}(k)=-\mu^{(+)} \pm \sqrt{\lambda_{+}(k l, n) \varepsilon_{0}^{2}+\left(\Delta^{(-)}\right)^{2}}, \\
& \text { where } \lambda_{+}(k l, n) \geq 1,
\end{aligned}
$$

where $n=1,2, \ldots$ is an index that labels different branches of solutions. By making use of the asymptotic behavior of the parabolic cylinder functions (see Eq. 9.246.2 in Ref. 52), one can show that $\lambda_{+}(k l, n) \simeq n$ when $k l>1$. This is expected since large values of $k l$ correspond to the states in the bulk, whose wave functions are localized around $\xi \simeq 0$ or equivalently $y / l \simeq k l$. (In a system without edges, index $n$ is identified with the usual Landau level index.)

Similarly, we can constrain the form of the spectrum in valley $K_{-}$(solutions of type II). The necessary condition for having a real solution to Eq. (36) is $\lambda_{-} \geq 0$. Thus, the energy spectrum in valley $K_{-}$has the following general structure:

$$
\begin{gathered}
E_{n}(k)=-\mu^{(-)} \pm \sqrt{\lambda_{-}(k l, n) \varepsilon_{0}^{2}+\left(\Delta^{(+)}\right)^{2}}, \\
\text { where } \lambda_{-}(k l, n) \geq 0,
\end{gathered}
$$

where $n=0,1,2, \ldots$ Again, one can show that $\lambda_{-}(k l, n) \simeq n$ when $k l \gg 1$.

Our numerical results for $\lambda_{ \pm}$as functions of $k l$ are presented in Fig. 3. Solid and dashed lines represent $\lambda_{+}$and $\lambda_{-}$, respectively. As expected, there exists an infinite tower of solutions that correspond to an infinite tower of Landau levels on a half-plane. In Fig. 3, we show only the first eleven solutions. In the same figure, we also added the constant solution $\lambda_{+}=0$ that, strictly speaking, represents only the dispersionless mode with the energy $E=-\mu^{(+)}+\Delta^{(-)}$, see the first expression in Eq. (38). (Formally, $\lambda_{+}=0$ may also mean that $E=-\mu^{(+)}-\Delta^{(-)}$, but this is not an energy eigenvalue.)

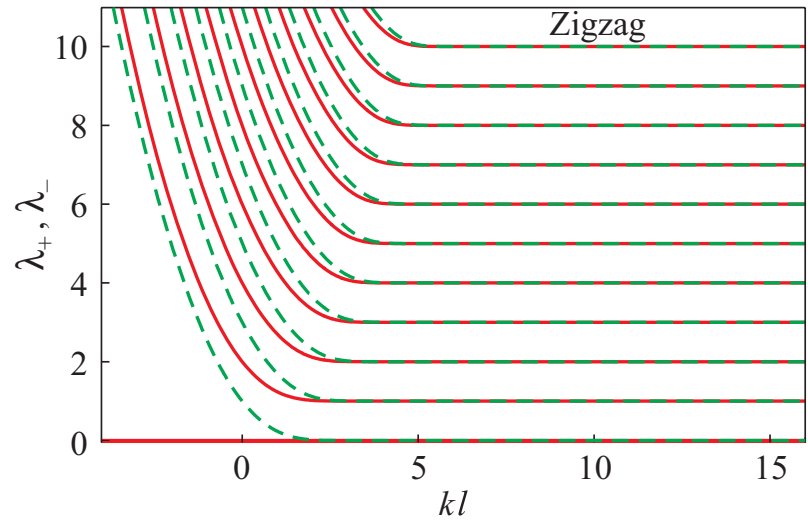

Fig. 3. The numerical solutions of Eq. (34) for the dimensionless parameter $\lambda_{+}$(solid line) and Eq. (36) for the dimensionless parameter $\lambda_{-}$(dashed line) in the case of a zigzag boundary. The solid line at $\lambda_{+}=0$ corresponds only to $E=$ $=-\mu^{(+)}+\Delta^{(-)}$solution.

By analyzing the structure of the spectrum together with the actual dependence of $\lambda_{ \pm}$on the wavevector, we can now determine when gapless modes exist in the spectrum of graphene on a half-plane with a zigzag edge. From Eqs. (38) and (39), we see that the necessary condition to have a zero energy state is that at least one of the following inequalities is satisfied:

$$
\begin{aligned}
& K_{+} \text {valley: }\left|\mu^{(+)}\right| \geq \sqrt{\varepsilon_{0}^{2}+\left(\Delta^{(-)}\right)^{2}}, \\
& K_{-} \text {valley: }\left|\mu^{(-)}\right| \geq\left|\Delta^{(+)}\right|
\end{aligned}
$$

From the fact that there exist branches with $\lambda_{+} \simeq 1$ and $\lambda_{-} \simeq 0$ at $k l>>1$, we see that this is also the sufficient condition.

An important point to emphasize here is that nonzero masses do not prevent the existence of the gapless edge states when the absolute value of $\Delta^{(+)}$is less than the absolute value of $\mu^{(-)}$at least for one choice of the spin. Our results generalize the findings of previous studies on a half-plane [17,23], where only the case with a single nonzero order parameter (either mass or spin gap) was considered.

Two specific examples of energy spectra, with and without gapless modes, are given in Fig. 4. In the left panel, the first few Landau levels in the case of a small spin gap, modeled by $\mu_{ \pm}=\mp 0.02 \varepsilon_{0}$ with the subscript index denoting the spin, and a larger singlet mass, given by $\Delta_{ \pm}= \pm 0.08 \varepsilon_{0}$, are shown. Since $\left|\mu^{(-)}\right|<\left|\Delta^{(+)}\right|$, there are no gapless modes in this case. In the right panel of Fig. 4 , the low-energy spectrum is shown for another choice of parameters, i.e., $\mu_{ \pm}=\mp 0.08 \varepsilon_{0}$ and $\Delta_{ \pm}= \pm 0.02 \varepsilon_{0}$, that satisfies the condition in Eq. (41). As expected, in this case there are gapless edge states in the spectrum. By taking into account that the group velocities of gapless modes, $v_{x}=\partial E /\left.\partial k\right|_{E=0}$, have opposite signs along the $x$ direction, the up- and down-spin states carry counter- 

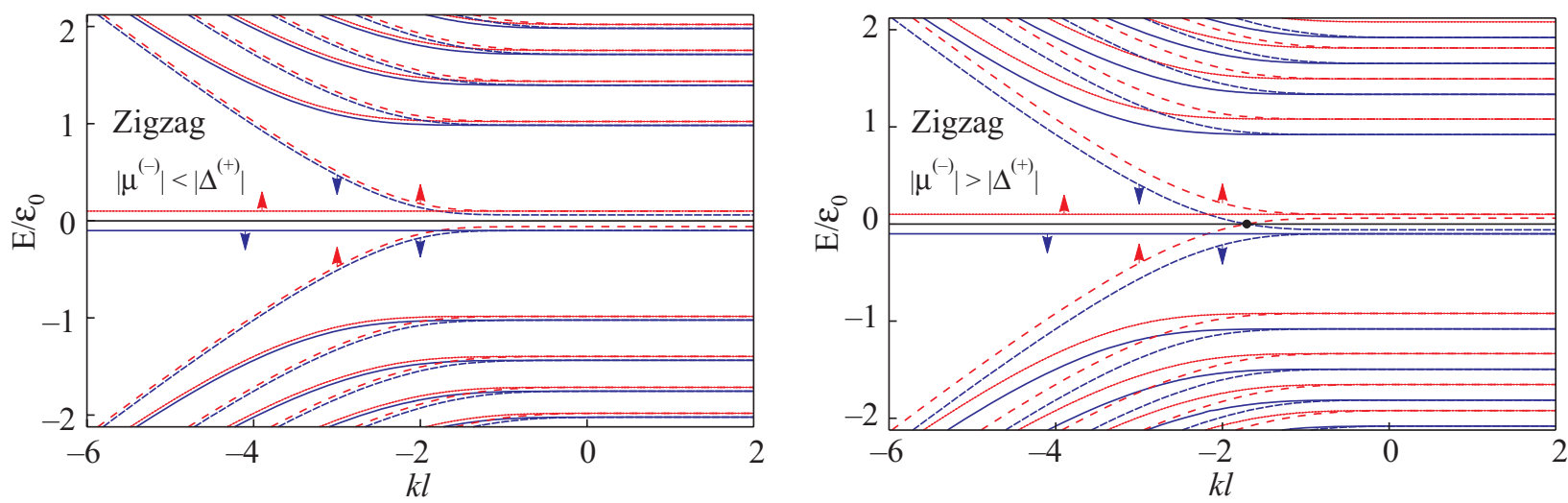

Fig. 4. Numerical results for the energy spectra of the first few Landau levels near a zigzag edge of graphene in the case of nonzero spin splitting and nonzero singlet masses. The values of parameters are $\mu_{ \pm}=\mp 0.02 \varepsilon_{0}$ and $\Delta_{ \pm}= \pm 0.08 \varepsilon_{0}$ in the left panel, and $\mu_{ \pm}=\mp 0.08 \varepsilon_{0}$ and $\Delta_{ \pm}= \pm 0.02 \varepsilon_{0}$ in the right panel. (The subscript indices in $\mu_{ \pm}$and $\Delta_{ \pm}$denote the spin orientations.) In the first case $\left|\mu^{(-)}\right|<\left|\Delta^{(+)}\right|$and there are no gapless modes, in the second case $\left|\mu^{(-)}\right|>\left|\Delta^{(+)}\right|$and gapless modes are present. Spin-up and spin-down states are denoted by red $(s=+)$ and blue $(s=-)$ lines (color online). In the lowest energy sublevels the spins are also marked by arrows. The spectra around $K_{+}\left(K_{-}\right)$point are shown by solid (dashed) lines.

propagating currents $[17,23]$. It is also curious to note that these gapless states are chiral since they belong to a single valley $\left(K_{-}\right)$.

Before concluding this section, it might be appropriate to mention that the examples of spectra shown in Fig. 4 may have a direct application to the case of graphene in a strong magnetic field. The corresponding choice of parameters with singlet, rather than triplet masses were taken in the same form as in the ground state around the neutral Dirac point, proposed in the dynamical model of Ref. 36. In fact, the spectra would look nearly the same also in the case of triplet masses, except perhaps for an overall shift of the dispersionless modes which depend not only on the absolute value but also on the sign of the mass terms.

\section{Edge states for a ribbon with zigzag edges}

In this section we study graphene ribbon of a finite width in the $y$ direction, $0 \leq y \leq W$, and a zigzag edge pa-

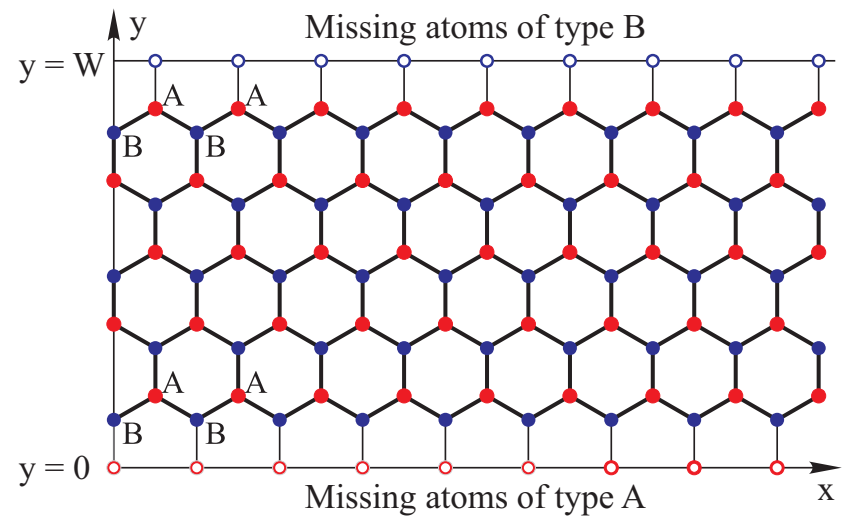

Fig. 5. The lattice structure of a finite width graphene ribbon. rallel to the $x$ direction, see Fig. 5 [37]. In this case, the $A$ and $B$ components of wave functions should vanish on the opposite edges $[23,45,47]$. Therefore, in addition to the boundary conditions at $y=0$ in Eq. (26), one should require that

$$
v_{+}(y=W)=v_{-}(y=W)=0 .
$$

In principle, by satisfying Eq. (26) and (42) and using the wave function normalization conditions, we can determine all four integration constants in Eqs. (27), (28), (30), and (31). For our purposes here, however, it suffices to determine the conditions when nontrivial, normalizable solutions exist. These will provide the dispersion spectra of all modes on a graphene ribbon.

Let us start from the boundary conditions for $u_{+}$and $v_{+}$components ( $K_{+}$valley), see Eqs. (26) and (42). They take the following explicit form:

$$
\begin{aligned}
& C_{1} \frac{E+\mu^{(+)}-\Delta^{(-)}}{\varepsilon_{0}} U\left(\frac{1-2 \lambda_{+}}{2},-\sqrt{2} k l\right)+ \\
& +C_{2} V\left(\frac{1-2 \lambda_{+}}{2},-\sqrt{2} k l\right)=0, \\
& C_{1} U\left(-\frac{1+2 \lambda_{+}}{2}, \sqrt{2}\left(k_{0}-k\right) l\right)+ \\
& +C_{2} \frac{E+\mu^{(+)}+\Delta^{(-)}}{\varepsilon_{0}} V\left(-\frac{1+2 \lambda_{+}}{2}, \sqrt{2}\left(k_{0}-k\right) l\right)=0,
\end{aligned}
$$

where $k_{0} \equiv W / l^{2}$ is determined by the width of the ribbon. A nontrivial solution to this set of equations exists when

$$
\lambda_{+} U\left(\frac{1-2 \lambda_{+}}{2},-\sqrt{2} k l\right) V\left(-\frac{1+2 \lambda_{+}}{2}, \sqrt{2}\left(k_{0}-k\right) l\right)-
$$



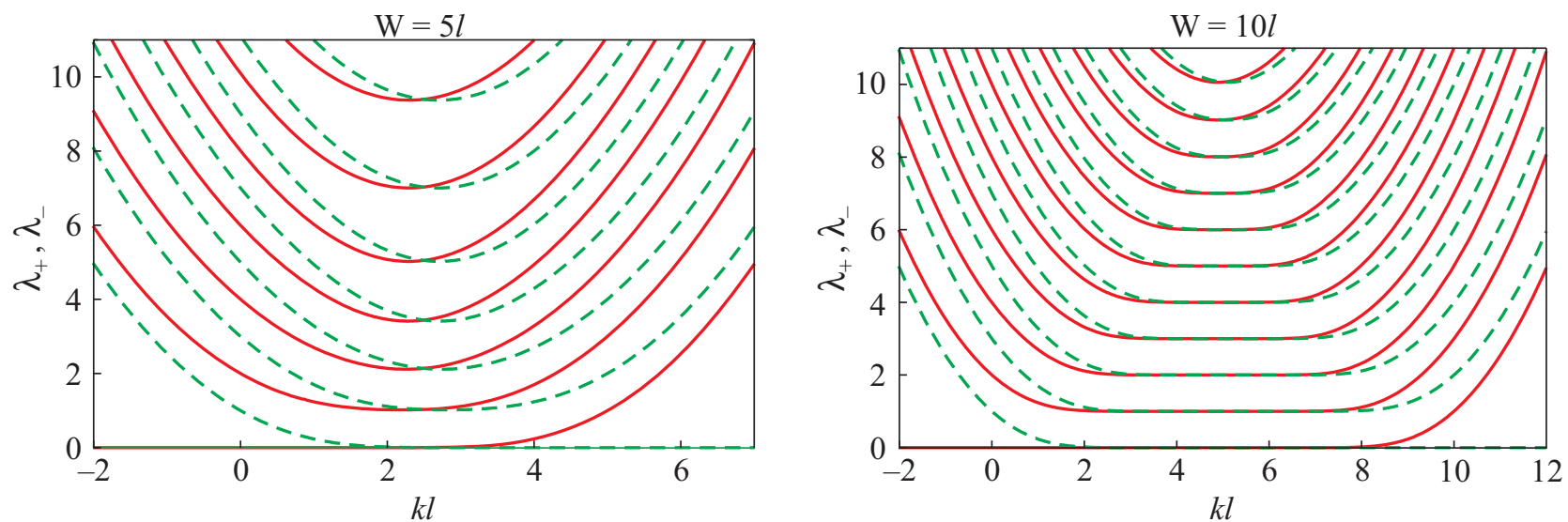

Fig. 6. Numerical results for the dimensionless parameters $\lambda_{+}$(solid lines) and $\lambda_{-}$(dashed lines) for two ribbons of widths $W=5 l$ (left panel) and $W=10 l$ (right panel).

$-U\left(-\frac{1+2 \lambda_{+}}{2}, \sqrt{2}\left(k_{0}-k\right) l\right) V\left(\frac{1-2 \lambda_{+}}{2},-\sqrt{2} k l\right)=0$.

Similar condition is also derived in the $K_{-}$valley,

$$
\begin{aligned}
& \lambda_{-} U\left(\frac{1-2 \lambda_{-}}{2}, \sqrt{2}\left(k_{0}-k\right) l\right) V\left(-\frac{1+2 \lambda_{-}}{2},-\sqrt{2} k l\right)- \\
& -U\left(-\frac{1+2 \lambda_{-}}{2},-\sqrt{2} k l\right) V\left(\frac{1-2 \lambda_{-}}{2}, \sqrt{2}\left(k_{0}-k\right) l\right)=0
\end{aligned}
$$

By solving Eqs. (45) and (46) numerically, we determine the dependence of dimensionless energy parameters $\lambda_{+}$ and $\lambda_{-}$on wavevector $k$. The results for two different widths of graphene ribbons, $W=5 l$ and $W=10 l$, are shown in Fig. 6.

When the width of the ribbon is less than about 3 or 4 times the magnetic length, we find that the spectra have little overlap with the usual bulk spectra, i.e., $\lambda_{ \pm}=n$ where $n$ is a nonnegative integer. Additionally, the separation between the nearest levels quickly increases with decreasing $W$. For example, in the case of $W=l$, the separation between the lowest two values of $\lambda_{ \pm}$is about an order of magnitude larger than in the bulk.

For the case $W=5 l$, shown in the left panel of Fig. 6, only the lowest level may have a hint at the middle plateau developing. However, when the ribbon's width is larger than about 6 or 7 times the magnetic length, nearly flat plateaus are already distinguishable in the lowest levels around the central wavevector $1 / 2 k_{0}$. We also find that the lower the level, the wider the plateau is formed. For example, in the case of $W=10 l$, about 8 or 9 lowest levels reveal clear plateaus, and the lowest of them in the middle are essentially indistinguishable from the levels that would appear in an infinite graphene sample, see the right panel in Fig. 6.

In passing, let us also emphasize the following special feature of the spectrum in a graphene ribbon with zigzag boundaries. As we see from Fig. 6 , for $\lambda_{+} \simeq 0$ (actually, $\left.E \simeq-\mu^{(+)}+\Delta^{(-)}\right)$and for $\lambda_{-} \simeq 0$ (actually, $E \simeq-\mu^{(-)}+$ $\left.+\Delta^{(+)}\right)$, dispersionless surface solutions [23,44,47] exist at both $K$ points. These solutions are bound to the $k \simeq 0$ and $k \simeq k_{0}$ edges for $K_{+}$and $K_{-}$points, respectively.

Now, by restoring the spin index, we assemble the complete spectrum of a graphene ribbon,

$$
\begin{aligned}
& E_{s, K_{+}}(n, k)=-\mu_{s}^{(+)} \pm \sqrt{\lambda_{+}(n, k) \varepsilon_{0}^{2}+\left(\Delta_{s}^{(-)}\right)^{2}} \\
& E_{s, K_{-}}(n, k)=-\mu_{s}^{(-)} \pm \sqrt{\lambda_{-}(n, k) \varepsilon_{0}^{2}+\left(\Delta_{s}^{(+)}\right)^{2}}
\end{aligned}
$$

Notice that for each spin orientation there are four sublevels that correspond to the lowest Landau level. Only half of these correspond to the «bulk» states that remain normalizable in an infinite graphene plane. The other half have wave functions localized only at the edges.

By making use of the numerical results for $\lambda_{+}$and $\lambda_{-}$, we can plot the actual energy spectra in the system. For the ribbons of widths $W=5 l$ and $W=10 l$, these are presented in Figs. 7 and 8. The choice of the order parameters in Fig. 7 is $\mu_{ \pm}=\mp 0.08 \varepsilon_{0}, \widetilde{\mu}_{ \pm}=0.01 \varepsilon_{0}, \Delta_{ \pm}= \pm 0.02 \varepsilon_{0}$, $\widetilde{\Delta}_{ \pm}=0$. In this case, the ferromagnetic gap $\mu_{s}^{( \pm)}$dominates over the mass gap $\Delta_{S}^{( \pm)}$, ensuring the presence of gapless edge states (marked by dots). The electron spins of the lowest energy sublevels are marked by arrows. In order to lift the degeneracy of all sublevels, we also added small nonzero values of $\tilde{\mu}_{ \pm}$. In Fig. 8 the parameters are chosen as follows: $\mu_{ \pm}=\mp 0.02 \varepsilon_{0}, \widetilde{\mu}_{ \pm}=0.01 \varepsilon_{0}, \Delta_{ \pm}=$ $= \pm 0.08 \varepsilon_{0}, \widetilde{\Delta}_{ \pm}=0$. There are no gapless edge states in this case.

From Figs. 7 and 8, we see that there exist gapless edge states (whose energy vanishes at certain values of $k$ ) only when the ferromagnetic gap dominates over the mass gap, i.e., $\left|\mu^{( \pm)}\right|>\left|\Delta^{(\mp)}\right|$. From Fig. 6, we can see that $\lambda_{ \pm}(0, k)$ is nonnegative and approaches zero at certain 

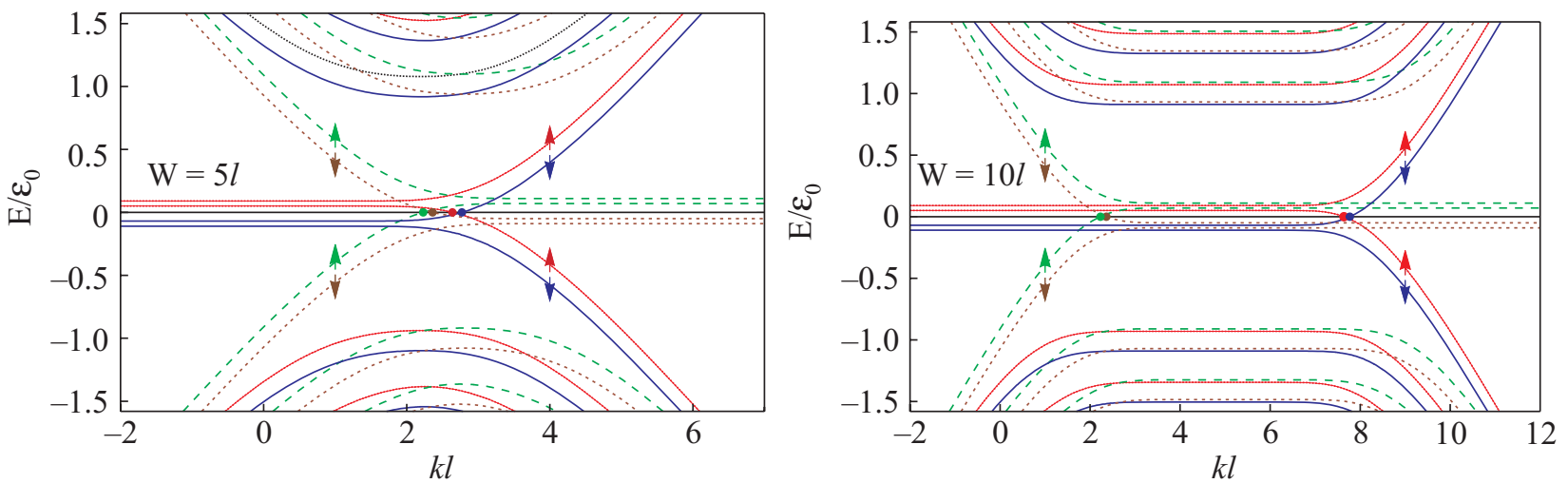

Fig. 7. Numerical results for the low-energy spectra for two ribbons of widths $W=5 l$ (left panel) and $W=10 l$ (right panel). The dynamical masses and ferromagnetic gaps are as follows: $\mu_{ \pm}=\mp 0.08 \varepsilon_{0}, \widetilde{\mu}_{ \pm}=0.01 \varepsilon_{0}, \Delta_{ \pm}= \pm 0.02 \varepsilon_{0}, \widetilde{\Delta}_{ \pm}=0$. In the lowest energy sublevels the spins are marked by arrows. The spectra around $K_{+}\left(K_{-}\right)$point are shown by solid (dashed) lines.

values of the wavevector. This feature together with dispersion relations (47) and (48) makes it clear that the necessary and sufficient condition for the existence of gapless modes is that at least one of the inequalities $\left|\mu_{s}^{(-)}\right|>\left|\Delta_{s}^{(+)}\right|,\left|\mu_{s}^{(+)}\right|>\left|\Delta_{s}^{(-)}\right|$is satisfied for at least one spin choice $s= \pm$.

These results for a finite width ribbon are similar to those on a half-plane given in the preceeding section. The only qualitative difference is due to the fact that on a ribbon there are additional edge states associated with the second edge which was absent in the case of a half-plane.

\section{Edge states for a half-plane with an armchair edge}

In this section, we analyze the spectrum of edge modes in the case of an armchair edge [38]. We take the armchair edge parallel to the $y$ direction, as shown in Fig. 2. In this case, it is convenient to use a different Landau gauge with $\left(A_{x}, A_{y}\right)=(0, B x)$. Accordingly, the solutions of Eq. (21) are translation invariant along the $y$ direction:

$$
\begin{aligned}
& \psi_{A K_{+}}(\mathbf{r}, k)=\frac{1}{\sqrt{2 \pi l}} \mathrm{e}^{i k y} u_{+}(x, k), \\
& \psi_{B K_{+}}(\mathbf{r}, k)=\frac{1}{\sqrt{2 \pi l}} \mathrm{e}^{i k y} v_{+}(x, k), \\
& \psi_{A K_{-}}(\mathbf{r}, k)=\frac{1}{\sqrt{2 \pi l}} \mathrm{e}^{i k y} u_{-}(x, k), \\
& \psi_{B K_{-}}(\mathbf{r}, k)=\frac{1}{\sqrt{2 \pi l}} \mathrm{e}^{i k y} v_{-}(x, k) .
\end{aligned}
$$

Then the corresponding differential equations for functions $u_{ \pm}(x, k)$, rewritten in terms of the dimensionless variable $\xi=x / l+k l$, coincide with Eq. (23). The expressions for the eliminated components $v_{ \pm}(\xi)$, however, slightly differ from Eq. (24), and are given by

$$
v_{ \pm}(\xi)=\mp i \frac{\varepsilon_{0}\left(\partial_{\xi} \mp \xi\right) u_{ \pm}(\xi)}{\sqrt{2}\left(E+\mu^{( \pm)} \mp \Delta^{(\mp)}\right)} .
$$

We consider a graphene sheet in the half-plane $x>0$. Since the armchair edge has lattice sites of both $A$ and $B$ type, the wave function should vanish at both these sites along the $x=0$ line $[23,45,47]$
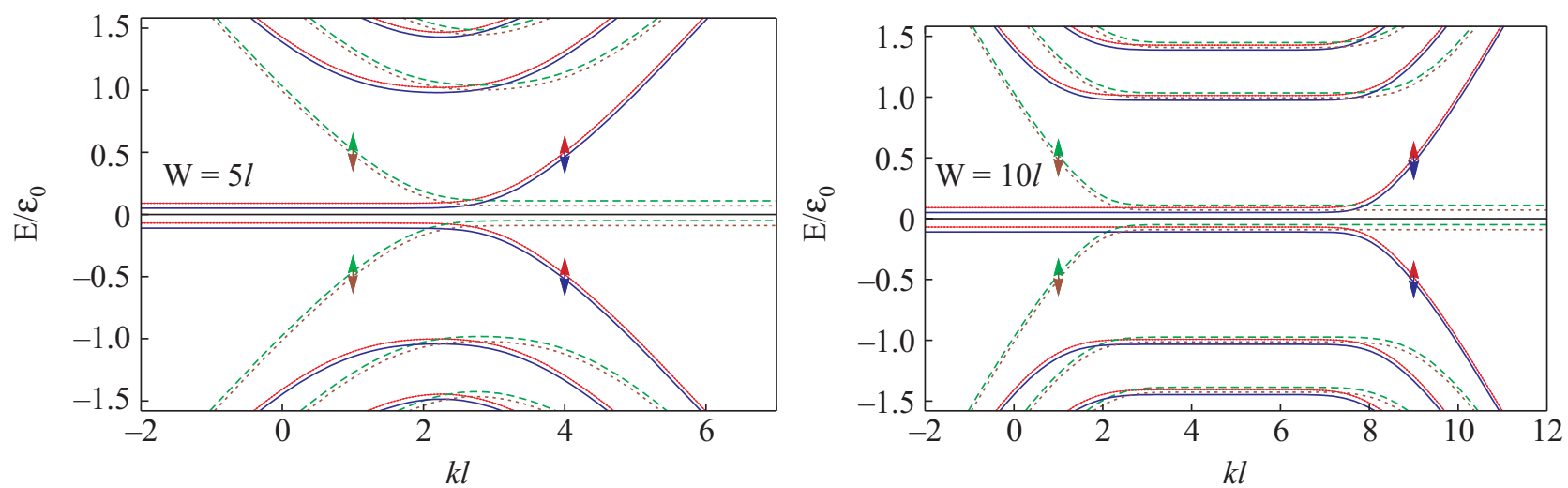

Fig. 8. Same as Fig. 7 but the dynamical masses and ferromagnetic gaps are as follows: $\mu_{ \pm}=\mp 0.02 \varepsilon_{0}, \widetilde{\mu}_{ \pm}=0.01 \varepsilon_{0}, \Delta_{ \pm}= \pm 0.08 \varepsilon_{0}$, $\widetilde{\Delta}_{ \pm}=0$. There are no gapless edge states in this case. 


$$
\begin{aligned}
& u_{+}(x=0)+u_{-}(x=0)=0, \\
& v_{+}(x=0)+v_{-}(x=0)=0 .
\end{aligned}
$$

Note that armchair boundary conditions mix the chiralities associated with $K_{+}$and $K_{-}$valleys. The general solutions for $u_{ \pm}(\xi)$ functions have the same form as in Eqs. (27) and (28),

$$
\begin{aligned}
& u_{+}(\xi)=C_{1} \frac{E+\mu^{(+)}-\Delta^{(-)}}{\varepsilon_{0}} U\left(\frac{1-2 \lambda_{+}}{2}, \sqrt{2} \xi\right)+ \\
& +C_{2} V\left(\frac{1-2 \lambda_{+}}{2}, \sqrt{2} \xi\right), \\
& u_{-}(\xi)=C_{3} U\left(-\frac{1+2 \lambda-}{2}, \sqrt{2} \xi\right)+ \\
& +C_{4} \frac{E+\mu^{(-)}+\Delta^{(+)}}{\varepsilon_{0}} V\left(-\frac{1+2 \lambda_{-}}{2}, \sqrt{2} \xi\right),
\end{aligned}
$$

but with $\xi=x / l+k l$. By using the relations in Eqs. (50) and (29), we also obtain the explicit expression for $v_{ \pm}(\xi)$ functions

$$
\begin{aligned}
& v_{+}(\xi)=i C_{1} U\left(-\frac{1+2 \lambda_{+}}{2}, \sqrt{2} \xi\right)+ \\
& +i C_{2} \frac{E+\mu^{(+)}+\Delta^{(-)}}{\varepsilon_{0}} V\left(-\frac{1+2 \lambda_{+}}{2}, \sqrt{2} \xi\right), \\
& v_{-}(\xi)=i C_{3} \frac{E+\mu^{(-)}-\Delta^{(+)}}{\varepsilon_{0}} U\left(\frac{1-2 \lambda_{-}}{2}, \sqrt{2} \xi\right)+ \\
& +i C_{4} V\left(\frac{1-2 \lambda_{-}}{2}, \sqrt{2} \xi\right) .
\end{aligned}
$$

As in the zigzag case, normalizable wave functions here are given in terms of only $U(a, z)$ function which falls off exponentially as $z \rightarrow+\infty$, unlike the function $V(a, z)$ which is growing exponentially in both directions $z \rightarrow \pm \infty$. Therefore, we set $C_{2}=0$ and $C_{4}=0$. Then, the armchair boundary conditions (51) lead to the following system of equations

$$
\begin{aligned}
& C_{1} \frac{E+\mu^{(+)}-\Delta^{(-)}}{\varepsilon_{0}} D_{\lambda_{+}-1}(\sqrt{2} k l)+C_{3} D_{\lambda_{+}}(\sqrt{2} k l)=0, \\
& C_{1} D_{\lambda_{-}}(\sqrt{2} k l)+C_{3} \frac{E+\mu^{(-)}-\Delta^{(+)}}{\varepsilon_{0}} D_{\lambda_{-}-1}(\sqrt{2} k l)=0,
\end{aligned}
$$

where again we used relation (33) to rewrite the expression in terms of the parabolic cylinder function $D_{v}(z)$. This system has nontrivial solutions when the determinant of coefficient functions is zero, i.e.,

$$
\begin{aligned}
& \frac{\left(E+\mu^{(+)}-\Delta^{(-)}\right)\left(E+\mu^{(-)}-\Delta^{(+)}\right)}{\varepsilon_{0}^{2}} \times \\
& \times D_{\lambda_{+}-1}(\sqrt{2} k l) D_{\lambda_{-}-1}(\sqrt{2} k l)-D_{\lambda_{+}}(\sqrt{2} k l) D_{\lambda_{-}}(\sqrt{2} k l)=0 .
\end{aligned}
$$

The numerical solutions to this equation for several representative choices of parameters are shown in Figs. 9 and 10 .

The two cases with singlet masses are illustrated in Fig. 9. In the left panel, the first few Landau levels in the case of $\mu_{ \pm}=\mp 0.02 \varepsilon_{0}$ and $\Delta_{ \pm}= \pm 0.08 \varepsilon_{0}$ are shown. In the right panel, instead, the corresponding values are $\mu_{ \pm}=\mp 0.08 \varepsilon_{0}$ and $\Delta_{ \pm}= \pm 0.02 \varepsilon_{0}$. Note that $\widetilde{\mu}_{ \pm}=\widetilde{\Delta}_{ \pm}=0$ in both cases. (Here we restored the subscript indices which denote the quasiparticle spin orientations.) As we see, in both cases the spectra contain gapless edge states. This is in strong contrast with the zigzag edge case. Indeed, for armchair edge gapless modes exist irrespective of the actual relation between the values of the singlet masses and spin splitting gaps. In part, this property could be understood from the topology of the spectra around the
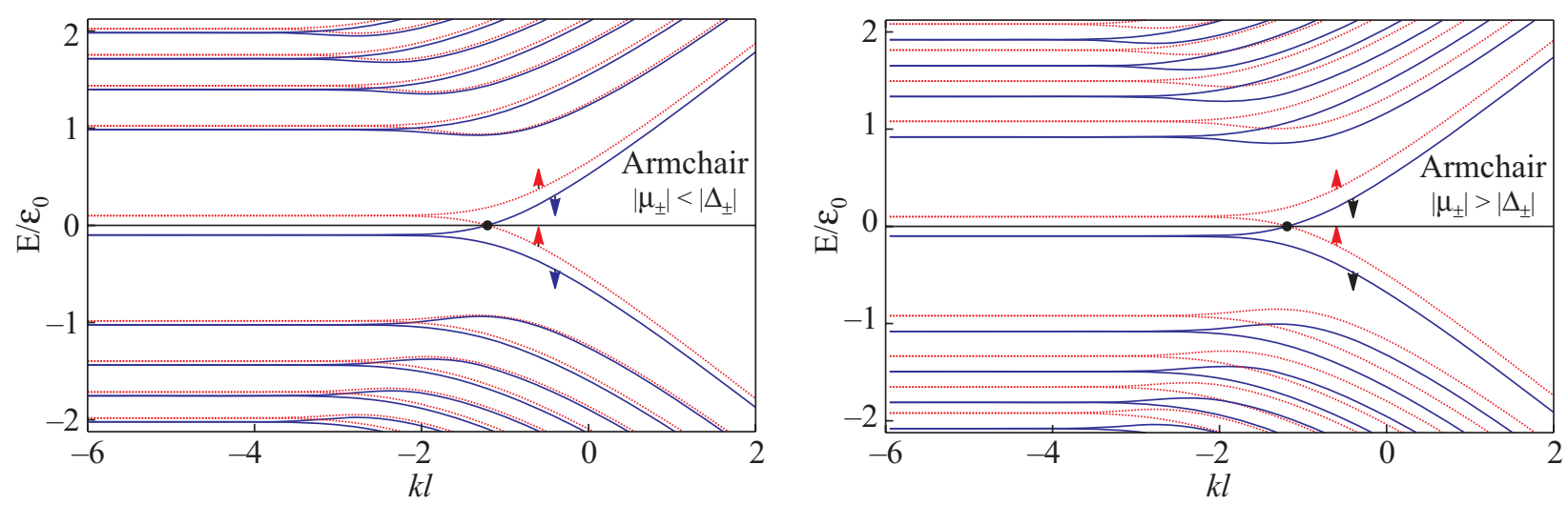

Fig. 9. Numerical results for the energy spectra of the first few Landau levels near a armchair edge of graphene in the case of nonzero spin splitting and nonzero singlet masses. The values of parameters are $\mu_{ \pm}=\mp 0.02 \varepsilon_{0}$ and $\Delta_{ \pm}= \pm 0.08 \varepsilon_{0}$ in the left panel, and $\mu_{ \pm}=\mp 0.08 \varepsilon_{0}$ and $\Delta_{ \pm}= \pm 0.02 \varepsilon_{0}$ in the right panel. (The subscript indices in $\mu_{ \pm}$and $\Delta_{ \pm}$denote the spin orientations.) In both cases, there are gapless modes in the spectrum. Spin-up and spin-down states are denoted by red $(s=+)$ and blue $(s=-)$ lines (color online). In the lowest energy sublevels the spins are also marked by arrows. 

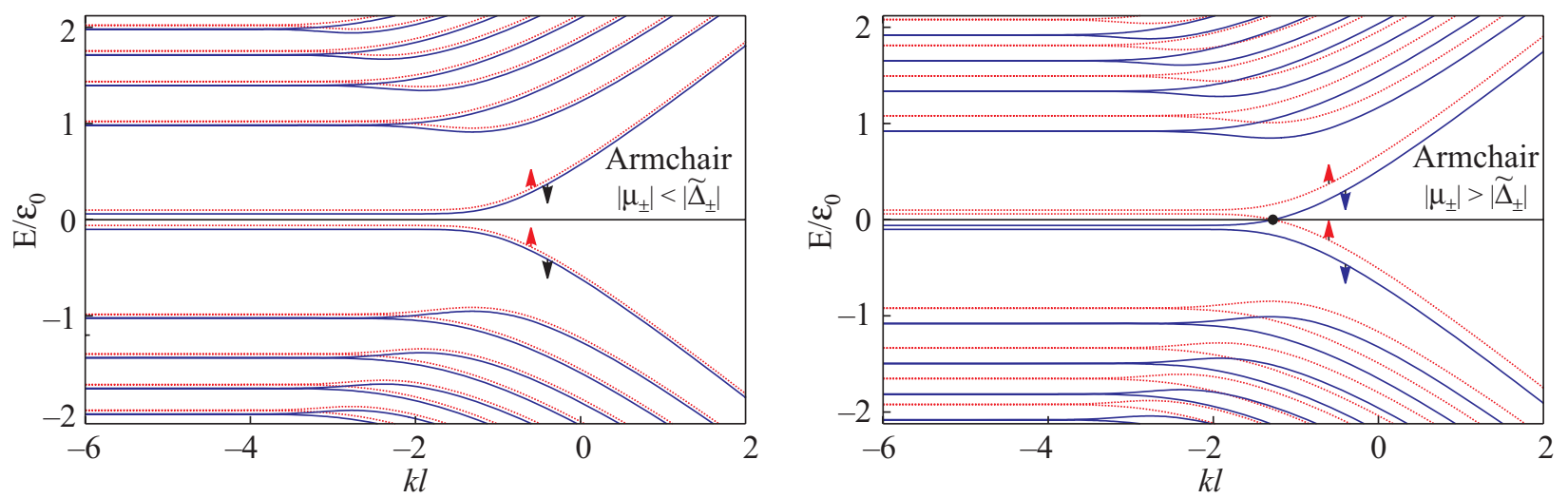

Fig. 10. Same as in Fig. 9, but for the case of nonzero triplet masses. The values of parameters are $\mu_{ \pm}=\mp 0.02 \varepsilon_{0}$ and $\widetilde{\Delta}_{ \pm}=0.08 \varepsilon_{0}$ in the left panel, and $\mu_{ \pm}=\mp 0.08 \varepsilon_{0}$ and $\widetilde{\Delta}_{ \pm}=0.02 \varepsilon_{0}$ in the right panel. The existence of gapless modes depends on the relative magnitude of $\left|\mu_{ \pm}\right|$and $\left|\widetilde{\Delta}_{ \pm}\right|$.

edge and the fact that the singlet mass does not break the valley $S U(2)_{s}$ symmetry. The double degenerate sublevels with a given spin, which should exist in the bulk because of the $S U(2)_{s}$ symmetry, repel in opposite directions near the edge. Then, gapless modes become almost inevitable at the edge.

We note that the gapless edge states in Fig. 9 consist of a pair of opposite spin states, carrying counter-propagating currents along the edge. This is qualitatively the same situation as found in Ref. 23. Interestingly, though, if the values of singlet masses $\Delta_{+}$and $\Delta_{-}$had the same signs, the opposite spin states would carry currents in the same direction along the edge. The observational implications of this fact could be quite unusual. It is not clear, however, if such a state can be realized since the dynamical model of Ref. 36 indicates that singlet masses $\Delta_{+}$and $\Delta_{-}$ should have opposite signs in the ground state.

The two cases with triplet masses are illustrated in Fig. 10. The values of the parameters in these cases are (i) $\mu_{ \pm}=\mp 0.02 \varepsilon_{0}$ and $\widetilde{\Delta}_{ \pm}=0.08 \varepsilon_{0}$ (left panel in Fig. 10) and (ii) $\mu_{ \pm}=\mp 0.08 \varepsilon_{0}$ and $\widetilde{\Delta}_{ \pm}=0.02 \varepsilon_{0}$ (right panel in Fig. 10). These energy spectra resemble the spectra for the zigzag edge, studied in the two previous sections. There are no gapless edge states when the mass is larger than the spin splitting, and there are such states when the mass is smaller than the spin splitting.

In fact, in the case of the triplet mass $\widetilde{\Delta}$ and a nonzero $\mu$ (but vanishing $\tilde{\mu}$ and $\Delta$ ), we can study the energy spectra around the armchair edge in a general case, just like we did for the zigzag edge. In this particular case, the spectral equation (57) takes the following simple form:

$$
\lambda D_{\lambda-1}^{2}(\sqrt{2} k l)-D_{\lambda}^{2}(\sqrt{2} k l)=0,
$$

where $\lambda=\left[(E+\mu)^{2}-\widetilde{\Delta}^{2}\right] / \varepsilon_{0}^{2}$. By expressing $\lambda$ in terms of squares of parabolic cylinder functions from Eq. (58), we see that solutions to this equation exist only with $\lambda \geq 0$. Therefore, the energy spectrum takes the form:
$E_{n}(k)=-\mu \pm \sqrt{\lambda(k l, n) \varepsilon_{0}^{2}+\widetilde{\Delta}^{2}}$, where $\lambda(k l, n) \geq 0$,

and $n=0,1,2, \ldots$ Additionally, one can show that $\lambda(k l, n) \simeq n$ when $|k l|>>1$ and $k$ is negative. Our numerical results for $\lambda$ as a function of $k l$ are presented in Fig. 11. Combining the numerical information with the general expression for the energy (59), we see that the necessary and sufficient condition for having gapless gapless modes is $|\mu| \geq \mid \widetilde{\Delta}$.

\section{Discussion}

Here we studied the spectra of edge states in graphene on a half-plane with zigzag and armchair boundary conditions, as well as on a finite width ribbon with zigzag edges. We derived the conditions for the existence of the gapless edge states for various types of masses and chemical potentials that could be generated spontaneously in QHE, e.g., at $v=0$ and $v= \pm 1$ plateaus.

Our analysis of singlet and triplet Dirac masses [with respect to the valley symmetry group $S U(2)_{S}$ ] shows that

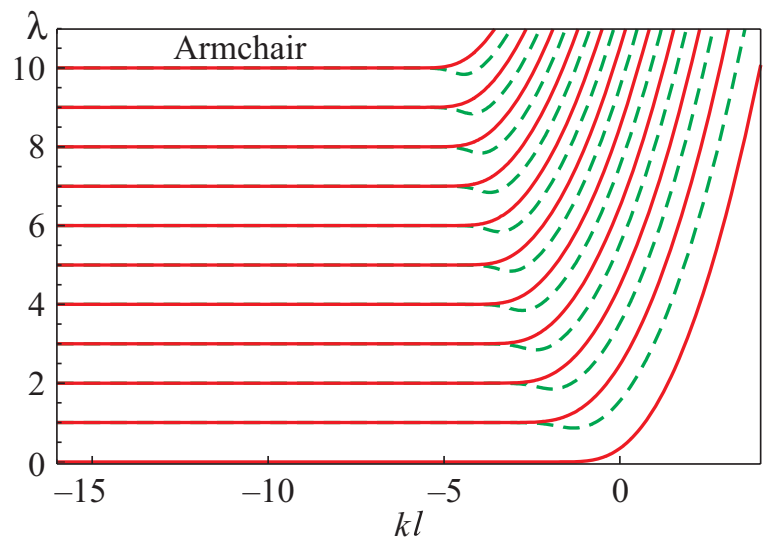

Fig. 11. Numerical solutions of Eq. (58) for the dimensionless parameter $\lambda$ in the case of an armchair boundary. This is valid for a general choice of $\widetilde{\Delta}$ and $\mu$, but only if $\widetilde{\mu}$ and $\Delta$ vanish. 
spectral properties of zigzag and armchair edges are affected by (i) the relative magnitude of the masses and chemical potentials, and (ii) the type of masses. In particular, we found the criteria for the existence of gapless edge states in the spectra. These can be summarized as follows [37,38].

(i) Zigzag edge: the necessary and sufficient condition to have gapless state is that at least one of the following inequalities is satisfied:

$$
\begin{aligned}
& \left|\mu_{s}^{(+)}\right| \geq \sqrt{\varepsilon_{0}^{2}+\left(\Delta_{s}^{(-)}\right)^{2}}, \\
& \left|\mu_{s}^{(-)}\right| \geq\left|\Delta_{s}^{(+)}\right| .
\end{aligned}
$$

On a finite width ribbon, both conditions take the same qualitative form, i.e., $\left|\mu_{s}^{( \pm)}\right| \geq\left|\Delta_{s}^{(\mp)}\right|$. This is because of the symmetry connecting two different zigzag edges with two different valleys, seen in the analysis.

(ii) Armchair edge:

a) gapless edge states exist always when there are singlet Dirac masses, irrespective of the actual relation between the values of the masses and the chemical potentials;

b) in the case of triplet Dirac masses, gapless edge states exist if $\left|\mu_{ \pm}\right|>\left|\widetilde{\Delta}_{ \pm}\right|$, and do not exist otherwise.

These conditions are consistent with the two limiting cases, analyzed in Ref. 23. The situation on a half-plane with a zigzag edge is essentially the same as on a ribbon, modulo the fact that there is one edge instead of two.

The results here are of interest in connection with the interpretation of the $v=0$ Hall plateau. Indeed, the gapless edge states should play an important role in charge transport of graphene in a strong magnetic field. Their presence is expected to make graphene a so-called quantum Hall metal, while their absence should make it an insulator $[17,23]$. The actual temperature dependence of the longitudinal resistivity at the $v=0$ plateau in Refs. 16, 17 is consistent with the metal type. This conclusion may be disputed in view of the recent data from Ref. 20 that reveal a clear plateau at $v=0$, but the temperature dependence of the diagonal component of the resistivity signals a crossover to an insulating state in high fields. The latter observations do not seem to support the existence of gapless edge states.

Our analysis of edge states [37,38] suggests that the conditions for the existence and absence of gapless edge states depend sensitively on the values of QHF and MC order parameters that characterize the nature of the corresponding QH state. Moreover, the microscopic analysis of Ref. 36 indicates that the order parameters of both types necessarily coexist. Therefore, the dynamics is very likely to be rich and full of surprises. The situation with the edge states is probably just one of such surprises.

\section{Acknowledgments}

The authors acknowledge useful discussions with E.V. Gorbar, H. Fertig, I.F. Herbut, M.I. Katsnelson, L.S. Levitov, and B.I. Shklovskii. V.P.G. and S.G. Sh. thank A.K. Geim for the discussion of the experimental data that indicate the existence of the gapless edge states in graphene. The work of V.P.G. was supported by the SCOPES project IB 7320-110848 of the Swiss NSF, the grant 10/07-N «Nanostructure systems, nanomaterials, nanotechnologies», the Program of Fundamental Research of the Physics and Astronomy Division of the National Academy of Sciences of Ukraine, and by the State Foundation for Fundamental Research of Ukraine (grant F16-457-2007). The work of V.A.M. was supported by the Natural Sciences and Engineering Research Council of Canada.

1. K.S. Novoselov, A.K. Geim, S.V. Morozov, D. Jiang, Y. Zhang, S.V. Dubonos, I.V. Grigorieva, and A.A. Firsov, Science 306, 666 (2004).

2. A.K. Geim and K.S. Novoselov, Nature Materials 6, 183 (2007).

3. A.H. Castro Neto, F. Guinea, N.M.R. Peres, K.S. Novoselov, and A.K. Geim, arXiv:0709.1163, submitted to Rev. Mod. Phys.

4. G.W. Semenoff, Phys. Rev. Lett. 53, 2449 (1984).

5. D.P. DiVincenzo and E.J. Mele, Phys. Rev. B29, 1685 (1984).

6. F.D.M. Haldane, Phys. Rev. Lett. 61, 2015 (1988).

7. G.P. Mikitik and Yu.V. Sharlai, Phys. Rev. Lett. 82, 2147 (1999).

8. S.G. Sharapov, V.P. Gusynin, and H. Beck, Phys. Rev. B69, 075104 (2004).

9. V.P. Gusynin and S.G. Sharapov, Phys. Rev. B71, 125124 (2005).

10. Y. Zheng and T. Ando, Phys. Rev. B65, 245420 (2002).

11. V.P. Gusynin and S.G. Sharapov, Phys. Rev. Lett. 95, 146801 (2005).

12. V.P. Gusynin and S.G. Sharapov, Phys. Rev. B73, 245411 (2006).

13. N.M.R. Peres, F. Guinea, and A.H. Castro Neto, Phys. Rev. B73, 125411 (2006).

14. K.S. Novoselov, A.K. Geim, S.V. Morozov, D. Jiang, M.I. Katsnelson, I.V. Grigorieva, S.V. Dubonos, and A.A. Firsov, Nature 438, 197 (2005).

15. Y. Zhang, Y.-W. Tan, H.L. Störmer, and P. Kim, Nature 438, 201 (2005).

16. Y. Zhang, Z. Jiang, J.P. Small, M.S. Purewal, Y.-W. Tan, M. Fazlollahi, J.D. Chudow, J.A. Jaszczak, H.L. Störmer, and P. Kim, Phys. Rev. Lett. 96, 136806 (2006).

17. D.A. Abanin, K.S. Novoselov, U. Zeitler, P.A. Lee, A.K. Geim, and L.S. Levitov, Phys. Rev. Lett. 98, 196806 (2007).

18. Z. Jiang, Y. Zhang, Y. Tan, H. Störmer, and P. Kim, Phys. Rev. Lett. 99, 106802 (2007).

19. A.J.M. Giesbers, U. Zeitler, M.I. Katsnelson, L.A. Ponomarenko, T.M. Mohiuddin, and J.C. Maan, Phys. Rev. Lett. 99, 206803 (2007) 
20. J.G. Checkelsky, L. Li, and N.P. Ong, Phys. Rev. Lett 100, 206801 (2008).

21. V.P. Gusynin, V.A. Miransky, S.G. Sharapov, and I.A. Shovkovy, Phys. Rev. B74, 195429 (2006).

22. V.P. Gusynin, V.A. Miransky, and I.A. Shovkovy, Phys. Rev. Lett. 73, 3499 (1994); Phys. Rev. D52, 4718 (1995); Nucl. Phys. B462, 249 (1996).

23. D.A. Abanin, P.A. Lee, and L.S. Levitov, Phys. Rev. Lett. 96, 176803 (2006); Solid State Commun. 143, 77 (2007).

24. K. Yang, Solid State Commun. 143, 27 (2007).

25. K. Nomura and A.H. MacDonald, Phys. Rev. Lett. 96, $256602(2006)$

26. M.O. Goerbig, R. Moessner, and B. Douçot, Phys. Rev. B74, 161407(R) (2006).

27. J. Alicea and M.P.A. Fisher, Phys. Rev. B74, 075422 (2006).

28. In both cases, the $S U(4)$ symmetry is associated with spinpseudospin degrees of freedom, but while in graphene pseudospin is related to the sublattice index, in bilayer quantum Hall systems pseudospin is related to the layer index.

29. I.F. Herbut, Phys. Rev. Lett. 97, 146401 (2006); Phys. Rev. B75, 165411 (2007); ibid. 76, 085432 (2007).

30. J.-N. Fuchs and P. Lederer, Phys. Rev. Lett. 98, 016803 (2007).

31. M. Ezawa, J. Phys. Soc. Jpn. 76, 094701 (2007); Physica E40, 269 (2007).

32. D.V. Khveshchenko, Phys. Rev. Lett. 87, 206401 (2001); ibid. 87, 246802 (2001); H. Leal and D.V. Khveshchenko, Nucl. Phys. B687, 323 (2004); D.V. Khveshchenko and W.F. Shively, Phys. Rev. B73, 115104 (2006).

33. E.V. Gorbar, V.P. Gusynin, V.A. Miransky, and I.A. Shovkovy, Phys. Rev. B66, 045108 (2002).

34. E.V. Gorbar, V.P. Gusynin, V.A. Miransky, and I.A. Shovkovy, Phys. Lett. A313, 472 (2003).

35. Y. Kopelevich, V.V. Lemanov, S. Moehlecke, and J.H.S. Torres, Fiz. Tverd. Tela 41, 2135 (1999) [Phys. Solid State 41, 1959 (1999)]; H. Kempa, Y. Kopelevich, F. Mrowka, A. Setzer, J.H.S. Torres, R. Höhne, and P. Esquinazi, Solid State Commun. 115, 539 (2000); M.S. Sercheli, Y. Kopelevich, R.R. da Silva, J.H.S. Torres, and C. Rettori, Solid State Commun. 121, 579 (2002); Y. Kopelevich, J.C. Medina Pantoja, R.R. da Silva, F. Mrowka, and P. Esquinazi, Phys. Lett. A355, 233 (2006).
36. E.V. Gorbar, V.P. Gusynin, and V.A. Miransky, Fiz. Nizk. Temp. 34, 1007 (2008).

37. V.P. Gusynin, V.A. Miransky, S.G. Sharapov, and I.A. Shovkovy, arXiv:0801.0708 [cond-mat.mes-hall].

38. V.P. Gusynin, V.A. Miransky, S.G. Sharapov, and I.A. Shovkovy, Phys. Rev. B77, 205409 (2008).

39. V.P. Gusynin, S.G. Sharapov, and J.P. Carbotte, Int. J. Mod. Phys. B21, 4611 (2007).

40. Strictly speaking, in order to preserve the $S U(2)_{S}$ valley symmetry of the model, all three components of the triplet should be included in the Lagrangian density on equal footing. The ground state, however, will correspond to a specific "vacuum alignment», e.g., characterized by a nonvanishing vacuum expectation value of the operator $\overline{\Psi_{s}} \gamma^{3} \Psi_{s}$

41. G. Giovannetti, P.A. Khomyakov, G. Brocks, P.J. Kelly, and J. van den Brink, Phys. Rev. B76, 073103 (2007).

42. S.Y. Zhou, G.-H. Gweon, A.V. Fedorov, P.N. First, W.A. de Heer, D.-H. Lee, F. Guinea, A.H. Castro Neto, and A. Lanzara, Nature Materials 6, 770 (2007).

43. K. Nakada, M. Fujita, G. Dresselhaus, and M.S. Dresselhaus, Phys. Rev. B54, 17954 (1996).

44. M. Fujita, K. Wakabayashi, K. Nakada, and K. Kusakabe, J. Phys. Soc. Jpn. 65, 1920 (1996).

45. E. McCann and V.I. Fal'ko, J. Phys.: Condens. Matter 16, 2371 (2004).

46. C.L. Kane and E.J. Mele, Phys. Rev. Lett. 95, 146802 (2005); ibid. 95, 226801 (2005).

47. L. Brey and H.A. Fertig, Phys. Rev. B73, 235411 (2006); ibid. 73, 195408 (2006).

48. N.M.R. Peres, A.H. Castro Neto, and F. Guinea, Phys. Rev. B73, 241403 (2006).

49. J. Milton Pereira, F.M. Peeters, and P. Vasilopoulos, Phys. Rev. B75, 125433 (2007).

50. M. Abramowitz and I.A. Stegun, Handbook of Mathematical Functions With Formulas, Graphs, and Mathematical Tables, U.S. Goverment Printing Office, Washington D.C. (1972), p. 685.

51. H. Bateman and A. Erdelyi, Higher Transcendental Functions, Vol. II, McGraw-Hill book Co., New York (1953).

52. I.S. Gradshtein and I.M. Ryzhik, Table of Integrals, Series and Products, Academic Press, Orlando (1980). 